\title{
FUZZY ROUGH INFORMATION MEASURES AND THEIR APPLICATIONS
}

\author{
Seema Singh ${ }^{1}$, D.S. Hooda ${ }^{2}$, S.C. Malik ${ }^{3}$ \\ ${ }^{1 \& 3}$ Department of Statistics, M.D. University, Rohtak-124001, Haryana, India \\ ${ }^{2}$ Honorary Professor of Mathematics, G.J. University of Science and \\ Technology, Hisar- 125001, Haryana, India \\ Corresponding Author - D.S. Hooda
}

\begin{abstract}
The degree of roughness characterizes the uncertainty contained in a rough set. The rough entropy was defined to measure the roughness of a rough set. Though, it was effective and useful, but not accurate enough. Some authors use information measure in place of entropy for better understanding which measures the amount of uncertainty contained in fuzzy rough set. In this paper three new fuzzy rough information measures are proposed and their validity is verified. The application of these proposed information measures in decision making problems is studied and also compared with other existing information measures.
\end{abstract}

\section{KEYWORDS AND PHRASES}

Fuzzy Rough Set, Similarity Information Measure, Logarithmic Information Measure, Weighted Information Measure and Decision Making Problem

\section{INTRODUCTION}

The extension of crisp set theory to fuzzy set and rough set theories was developed by Zadeh [26] and Pawlak [16] respectively. People use to compare rough set with that of fuzzy set, but both the notions in aims and objectives are different. However, there is no sense to compare which one is better or more useful than other. Rough set theory has its own importance in artificial intelligence and in cognitive sciences, particularly in the areas of pattern recognition, machine learning, inductive reasoning, knowledge acquisition, etc.

The concept of rough set theory sometimes overlaps with Dempster-Shafer theory [23] of evidence. But the main difference between these is that the main tool in Dempster-Shafer theory is a belief function, where in rough set theory, lower and upper approximations sets are used. No preliminary and additional information is needed in rough set theory like membership grade in fuzzy set theory, probability distribution in statistics and basic probability assignment in Dempster-Shafer theory.

A rough set deals with incomplete information where a fuzzy set deals with vagueness, so it is interesting to know how to handle the real life problems having both incomplete information as well as vagueness in data. Thus, to handle such kind of situation Nakamura [13] and Dubois and Prade [4] introduced the concept of fuzzy rough set, which was called as a hybrid model of fuzzy and rough sets. Thereafter, Nanda and Majumdar [14] widely used this concept in the development of their research. Banerjee and Pal [1] studied the roughness of fuzzy set in 1996 and Verbiest [25] worked on fuzzy rough and evolutionary approaches to instance selection. 
Jabar and Rashid [9] combined fuzzy rough set with salient features for human resource management classification.

Some trigonometric information measures for fuzzy rough set and their applications in medical diagnosis were studied by Sharma and Gupta [21]. Similarity and distance information measures on fuzzy rough set with their applications were described by Sharma et al. [19]. Recently, a new approach to rough set based on remote system was introduced by Sun et al. [24]. Zhan et al. [28] also studied covering based multi-granulation (I,T)- fuzzy rough set models and their applications in multi-attribute group decision-making problems.

The word "entropy" was first used to measure an amount of uncertainty in probability distribution of a random variable in an experiment by Shannon [18]. Later, non-probabilistic entropy of a fuzzy set was proposed and described by Zadeh [27]. Fuzziness of a fuzzy set due to ambiguity, impreciseness and vagueness can be measured by using fuzzy entropy which was defined and characterized by De Luca and Termini [3].

Several other researchers, like Kapur [10], Liu [12], Pal and Pal [15], Kosko [11] and Gupta and Sheoran [5] used Shannon's entropy axioms of characterization to measure uncertainty in fuzzy rough set. A similarity information measure between fuzzy rough set and fuzzy rough values was defined by Chengyi et al. [2] and that was characterized by Qi \& Chengyi [17] in 2008.

Logarithmic entropy for fuzzy rough set and its application in decision making was proposed by Sharma [22]. The word entropy is a tedious word usually not understood easily, so fuzzy entropy was replaced by fuzzy information measure. Thus, some authors have called fuzzy entropy as fuzzy information measure. Hooda and Jain [6] in 2009 introduced three sub additive measures of fuzzy information and studied their applications in medical and social sciences. A new information measure of a fuzzy set was suggested and characterized by Hooda and Bajaj [7] and called it as "useful" fuzzy information measure. Hooda and Raich [8] unified existing work of various authors and described various generalizations of fuzzy information measures with their applications.

In the present paper, some new logarithmic information measures for fuzzy rough values and fuzzy rough set are proposed and their applications are studied. Basic concepts and definitions used in the later development of the paper are described in section 2. In section 3, a new logarithmic information measure for fuzzy rough values is defined and its validity is proved. Another information measure for fuzzy rough set and its application with illustrations are studied in section 4. In section 5, a weighted information measure for fuzzy rough set is discussed with its application. Comparison of the information measure with other existing information measures is studied in section 6. Conclusion is given in section 7 with references at the end of paper.

\section{Preliminaries}

In this section some basic concepts and definitions used in development of the later part of the paper are described and illustrated with examples.

\section{Definition 2.1[ 26 ]}

Let $X$ be a non-empty universal set and $A$ is a subset of $X$, then a function $\mu_{A}(x): X \rightarrow[0,1]$, defines fuzzy set on $\mathrm{X}$ and is usually written as

$$
A=\left\{\left(x_{i}, \mu_{A}\left(x_{i}\right): \mu_{A}\left(x_{i}\right) \in[0,1] ; \forall x_{i} \in X\right)\right\}
$$

where $\mu_{A}(x)$ is called membership function from $X$ to $[0,1]$ with the following properties: 


$$
\mu_{A}(x)=\left\{\begin{array}{lc}
0, & \text { if } x \notin A \text { there is no ambiguity } \\
1, & \text { if } x \in A \text { there is no ambiguity } \\
0.5, & \text { whether } x \in A \text { or } x \notin \text { A there is } \\
\text { maximum abiguity }
\end{array}\right.
$$

\section{Definition 2.2[4]}

Let $U$ be the universal set and $R$ be an equivalent relation on $U \times U$ which is also known as indistinguishable relation, then $W=(U, R)$ is known as Pawlak approximation space. The set of equivalent class generated by $R$ on $U$ i.e. $U / R=\left\{X_{1}, X_{2}, \ldots, X_{m}\right\}$ is called knowledge or equivalence class, $[x]_{R}$ is equivalent class of elements $x$ and the set of all fuzzy sets on $U$ is denoted by $F(U)$. Now suppose $\forall X \subseteq U, X=\{x \in U \mid[x] \subseteq X\}$ and $\bar{X}=\{x \in U \mid[x] \cap X \neq$ $\emptyset$, then $X=(\underline{X}, \bar{X})$ is called rough set in $W$ and $\underline{X}$ and $\bar{X}$ are called lower and upper approximation of $X$ on $\mathrm{W}$ respectively.

\section{Example2.1}

Let $\mathrm{U}=\{1,2,3,4\}$ be a universal set, $\mathrm{B}=\{\{1,2\},\{3,4\}\}$ and $\mathrm{X}=\{1,2,3\}(\mathrm{X} \subset \mathrm{U})$. Let $\mathrm{B}(\mathrm{X})$ be the rough set of $X$, then $B(X)=\{\{1,2\},\{1,2,3,4\}\}=\{\{1,2,3\},\{1,2,4\}\}$, where $\{1,2\}$ is the lower approximation of $X$ and $\{1,2,3,4\}$ is upper approximation of $X .\{\{1,2,3\},\{1,2,4\}\}$ is family of all sets containing $\{1,2\}$ and $\{1,2,3,4\}$ astheirlowerandupperapproximations. Thus, $(\{1,2\},\{1,2,3,4\})$ is the rough set of $\{1,2,3\}$.

\section{Definition 2.3[4]}

Let $U$ be universe of discourse, $R$ be a fuzzy relation on $U \times U$ and $(U, R)$ is fuzzy approximation space. For any set $A \in F(U)$,the lower and upper approximations of A namely $R(A)$ and $\overline{R(A)}$ with respect to approximation space $(U, R)$ are called fuzzy sets of $U$ whose membership functions are defined by

$$
\begin{gathered}
\underline{R(A)}=\vee_{y \in U}[R(x, y) \wedge A(y)], x \in U \text { and } \\
\overline{R(A)}=\wedge_{y \in U}[(1-R(x, y)) \vee A(y)], \quad x \in U .
\end{gathered}
$$

Hence, the pair $(R(A), \overline{R(A)})$ is defined as fuzzy rough set.

\section{Example2.2.}

Suppose $R=\left\{r_{1}, r_{2}, r_{3}, r_{4}, r_{5}\right\}$ be the set of objects and $C=\left\{c_{1}, c_{2}, c_{3}, c_{4}, c_{5}, c_{6}\right\}$ be the set of parameters, then fuzzy rough set $(\mathrm{R}, \mathrm{C})$ is given below: 
Table 2.1: Fuzzy Rough Set

\begin{tabular}{|c|c|c|c|c|c|c|}
\hline $\begin{array}{l}\mathbf{C} \\
\mathbf{R}\end{array}$ & $\boldsymbol{c}_{\mathbf{1}}$ & $\boldsymbol{c}_{\mathbf{2}}$ & $\boldsymbol{c}_{\mathbf{3}}$ & $\boldsymbol{c}_{\mathbf{4}}$ & $\boldsymbol{c}_{\mathbf{5}}$ & $\boldsymbol{c}_{\mathbf{6}}$ \\
\hline $\boldsymbol{r}_{\mathbf{1}}$ & $(.1, .5)$ & $(.2, .6)$ & $(.4, .8)$ & $(.7, .9)$ & $(.6, .8)$ & $(.4, .6)$ \\
\hline $\boldsymbol{r}_{\mathbf{2}}$ & $(.2, .3)$ & $(.1, .3)$ & $(.4, .9)$ & $(.6, .8)$ & $(.5, .6)$ & $(.8, .8)$ \\
\hline $\boldsymbol{r}_{\mathbf{3}}$ & $(.1, .5)$ & $(.3, .5)$ & $(.5, .7)$ & $(.3, .8)$ & $(.6, .6)$ & $(.4, .9)$ \\
\hline $\boldsymbol{r}_{\mathbf{4}}$ & $(.5, .7)$ & $(.1, .5)$ & $(.3, .8)$ & $(.3, .7)$ & $(.1, .3)$ & $(.5, .8)$ \\
\hline $\boldsymbol{r}_{\mathbf{5}}$ & $(.4, .6)$ & $(.5, .7)$ & $(.3, .5)$ & $(.7, .9)$ & $(.4, .5)$ & $(.5, .6)$ \\
\hline
\end{tabular}

\section{Definition 2.4[19]}

A real-valued function that enumerates the similarity between two objects is defined as similarity information measure. Actually, there is no particular definition of similarity information measures, in general these information measures are some implication of inverse of distance measures. Scores are given for similar quality, high score for more similar objects and low or negative scores for dissimilar quality.

Let $S$ be the similarity measure on $H$, then the entropy corresponding to $\mathrm{S}$ is as follows:

$$
e(F)=S\left(F, F^{C}\right), \forall F \in H,
$$

where ' $e$ ',the entropy on $H$ is the entropy generated by similarity information measure $S$ and denoted by $e(F)$.

\section{INFORMATION MEASURE FOR FuZZY ROUGH VALUES}

In this section a new information measure for fuzzy rough values is proposed on the lines of other existing similarity information measures.

\section{Definition 3.1[3]}

A real valued function $e: A \rightarrow[0,+\infty)$, is a fuzzy information measure on $A$ if $e$ satisfies the following four axioms $\forall x \& y \in A$ :

a) $e(x)=0$, if $x=[0,0]$ or $x=[1,1]$ i.e. $\underline{x} \& \bar{x}=0$ or 1 .

b) $e(x)=e\left(x^{C}\right)$.

c) Fuzzy Information measuree assumes a unique value that is $e(x)=1$ for $x=[0.5,0.5]$.

d) $e(x) \geq e(y)$, if $y$ is crisper than $x$, i.e. $x \geq y$ for $x \leq 0.5(\bar{x} \leq 0.5)$ and $y \geq x$ for $x \geq$ $0.5(\underline{x} \geq 0.5)$.

In 2004 Chengyi et al. [2] defined a similarity information measure between two fuzzy rough values which is as follows:

Let $\mathrm{A}$ be a fuzzy rough set and $\mathrm{x}, \mathrm{y}$ are the fuzzy rough values in $A$, then the degree of similarity between fuzzy rough values $\mathrm{x}$ and $\mathrm{y}$ is given by $M_{Z}$ as

$$
M_{Z}(\mathrm{x}, \mathrm{y})=1-\frac{1}{2}(|\underline{x}-\underline{y}|-|\bar{x}-\bar{y}|)
$$

A similarity information measure between fuzzy rough sets and its elements was defined by Qi and Chengyi [17] in 2008 as given below: 
Let $\mathrm{A}$ be a fuzzy rough and $\mathrm{x}, \mathrm{y}$ are the fuzzy rough values in $A$, then similarity degree between elements $\mathrm{x}$ and $\mathrm{y}$ can be evaluated as

$$
M(x, y)=1-\frac{1}{2}\left(\rho_{x y}+\sigma_{x y}\right)
$$

where $\rho_{x y}=\left|\rho_{x}-\rho_{y}\right|, \sigma_{x y}=\left|\sigma_{x}-\sigma_{y}\right|$ and $\tau_{x}=\bar{x}-\underline{x}$ is called the degree of indeterminacy of element $x \in A$. $\rho_{x}=\underline{x}+\tau_{x} \underline{x}=\left(1+\tau_{x}\right) \underline{x}$ and $\sigma_{x}=1-\bar{x}+\tau_{x}(1-\bar{x})$ are called degree of favour $x \in A$.

Sharma et al. [20] defined entropy for fuzzy rough values corresponding to similarity information measure (3.1) as follows:

$$
e(x)=1-\frac{1}{2}(|2 \underline{x}-1|+|2 \bar{x}-1|), \forall x \in A,
$$

where $\mathrm{A}$ is a fuzzy rough set and $x=(\underline{x}, \bar{x})$.

Corresponding to the similarity information measure (3.3), logarithmic information measure for fuzzy rough values is proposed as given below:

$$
\boldsymbol{e}_{\boldsymbol{l o g}}(\boldsymbol{x})=\log _{2}\left(2-\frac{1}{2}(|2 \underline{x}-1|+|2 \bar{x}-1|)\right), \forall x \in A,
$$

where $\mathrm{A}$ is a fuzzy rough set and $x=(\underline{x}, \bar{x})$.

(3.4) is called a similarity fuzzy rough information measure which must satisfy the four axioms given in definition (3.1) for its validity. Thus, these are verified by as lemmas 1 to 4 and explained below:

\section{Lemma1}

Let A be a fuzzy rough set then $\forall x \in A$ and $x=(\underline{x}, \bar{x})$, the fuzzy rough information measure defined in (3.4) is equal to zero for $x=[0,0]$ or $x=[1,1]$ i.e. $x=0$ or 1 and $\bar{x}=0$ or 1 .

\section{Proof}

Putting $x=[0,0]$ i.e. $\underline{x}=0$ and $\bar{x}=0$ in (3.4) we get

$$
\begin{gathered}
e_{\log }(x)=\log _{2}\left(2-\frac{1}{2}(|2 \times 0-1|+|2 \times 0-1|)\right) \\
\Rightarrow e_{\log }(x)=\log _{2}\left(2-\frac{1}{2}(|0-1|+|0-1|)\right) \\
\Rightarrow e_{\log }(x)=\log _{2}\left(2-\frac{1}{2} \times 2\right) \\
\Rightarrow e_{\log }(x)=\log _{2}(2-1)=\log _{2} 1=0 .
\end{gathered}
$$

Again putting $x=[1,1]$ i.e. $\cdot \underline{x}=1$ and $\bar{x}=1$ in (3.4) we get

$$
\begin{gathered}
e_{\log }(x)=\log _{2}\left(2-\frac{1}{2}(|2 \times 1-1|+|2 \times 1-1|)\right) \\
\Rightarrow e_{\log }(x)=\log _{2}\left(2-\frac{1}{2}(|2-1|+|2-1|)\right)
\end{gathered}
$$




$$
\begin{gathered}
\Rightarrow e_{\log }(x)=\log _{2}\left(2-\frac{1}{2} \times 2\right) \\
\Rightarrow e_{\log }(x)=\log _{2}(2-1)=\log _{2} 1=0 .
\end{gathered}
$$

Hence, for $x=[0,0]$ or $x=[1,1]$ the fuzzy rough information measure (3.4) is zero.

\section{Lemma2}

Let A be a fuzzy rough set then $\forall x \in A$ and $x=(\underline{x}, \bar{x})$, the fuzzy rough information measure defined in (3.4) is equal to its compliment.

\section{Proof}

The measured (3.4) is equal to its compliment when $e_{\log }\left(x^{C}\right)=e_{\log }(x)$, for this put $\underline{x}=1-$ $\underline{x}$ and $\bar{x}=1-\bar{x}$ in equation (3.4), we get

$$
\begin{gathered}
e_{\log }\left(x^{C}\right)=\log _{2}\left(2-\frac{1}{2}(|2(1-\underline{x})-1|+|2(1-\bar{x})-1|)\right) \\
\Rightarrow e_{\log }\left(x^{C}\right)=\log _{2}\left(2-\frac{1}{2}(|2-2 \underline{x}-1|+|2-2 \bar{x}-1|)\right), \\
\Rightarrow e_{\log }\left(x^{C}\right)=\log _{2}\left(2-\frac{1}{2}(|1-2 \underline{x}|+|1-2 \bar{x}|)\right), \\
\Rightarrow e_{\log }\left(x^{C}\right)=\log _{2}\left(2-\frac{1}{2}(|2 \underline{x}-1|+|2 \bar{x}-1|)\right) .
\end{gathered}
$$

Clearly, $e_{\log }\left(x^{C}\right)=e_{\log }(x)$, hence information measure (3.4) is equal to its compliment.

Thus, second condition is also satisfied.

\section{Lemma3}

Let $\mathrm{A}$ be a fuzzy rough set, then $\forall x \in A$ and $x=(\underline{x}, \bar{x} z z y)$, the fuzzy rough information measure defined in (3.4) is equal to one i.e. it assumes a unique maximum value for $x=$ $[0.5,0.5]$.

\section{Proof}

Let us put $\underline{x}=0.5$ and $\bar{x}=0.5$ in equation (3.4), then

$$
\begin{gathered}
e_{\log }(x)=\log _{2}\left(2-\frac{1}{2}(|2 \times 0.5-1|+|2 \times 0.5-1|)\right) \\
\Rightarrow e_{\log }(x)=\log _{2}\left(2-\frac{1}{2}(|1.0-1|+|1.0-1|)\right) \\
\Rightarrow e_{\log }(x)=\log _{2}\left(2-\frac{1}{2}(|0|+|0|)\right) \\
\Rightarrow e_{\log }(x)=\log _{2}(2-0)=\log _{2}(2)=1
\end{gathered}
$$

Thus, for $x=[0.5,0.5]$ fuzzy rough information measure (3.4) assumes a unique maximum value 1 . Hence third property is satisfied. 


\section{Lemma 4}

Let A be a fuzzy rough set, then $\forall x \in A$ and $x=(\underline{x}, \bar{x})$, we have $e_{\log }(x) \geq e_{\log }(y)$ if $y$ is sharper or crisper than $x$ i.e. $y \leq x$ for $x \leq 0.5(\bar{x} \leq 0.5)$ and $y \geq x$ for $x \geq 0.5(\underline{x} \geq 0.5)$.

\section{Proof}

For the first case let $y \leq x$ for $x \leq 0.5(\bar{x} \leq 0.5)$,

$$
\begin{gathered}
\Rightarrow \underline{y} \leq \bar{y} \leq \bar{x} \leq 0.5 \& \underline{y} \leq \underline{x} \leq \bar{x} \leq 0.5, \\
\Rightarrow|2 \bar{x}-1| \leq|2 \bar{y}-1| \text { and }|2 \underline{x}-1| \leq|2 \underline{y}-1|, \\
\Rightarrow \frac{1}{2}(|2 \bar{x}-1|+|2 \underline{x}-1|) \leq \frac{1}{2}(|2 \bar{y}-1|+|2 \underline{y}-1|) .
\end{gathered}
$$

Subtracting the equation on both side from ' 2 ' and taking binary logarithm we get

$$
\begin{gathered}
\log _{2}\left(2-\frac{1}{2}(|2 \bar{x}-1|+|2 \underline{x}-1|)\right) \geq \log _{2}\left(2-\frac{1}{2}(|2 \bar{y}-1|+|2 \underline{y}-1|)\right), \\
\Rightarrow e_{\log }(x) \geq e_{\log }(y) .
\end{gathered}
$$

Similarly, second case can be proved. Thus, all the four axioms given in definition (3.1) are satisfied by fuzzy rough information measure (3.4). Hence, it is a valid information measure.

\section{INFORMATION MEASURE FOR FUZZY ROUGH SETS}

In the previous section we have defined an information measure for fuzzy rough values. Next, corresponding to equation (3.4) another information measure for fuzzy rough set is proposed. Let us consider a fuzzy rough set ' $A$ ', then information measure for fuzzy rough set ' $A$ ' is proposed as given below:

$$
E_{\log }(A)=\frac{1}{n} \sum_{i=1}^{n} \log _{2}\left(2-\frac{1}{2}\left(\left|2 \underline{x_{i}}-1\right|+\left|2 \overline{x_{i}}-1\right|\right)\right), \forall x_{i} \in A \text { and } x_{i}=\left(\underline{x_{i}}, \overline{x_{i}}\right)
$$

(4.1) is called as a fuzzy rough information measure and it lies in the interval $[0,1]$. Larger value of the fuzzy rough information measure indicates more uncertainty in ' $A$ '. Obviously, four lemmas for the validity of the information measure hold.

Next, some propositions are enumerated as

\section{Proposition 4.1}

Let $A$ be a fuzzy rough set,then $\forall x_{i} \in A$ value of information measure (4.1) is equal to zero for $\underline{x_{i}}=0$ or 1 and $\overline{x_{i}}=0$ or 1 i.e. for A to be a crisp set.

\section{Proposition 4.2}

Let $A$ be a fuzzy rough set, then $\forall x_{i} \in A$ value of information measure (4.1) is equal to the value of its complement, where $A^{C}=\left(1-\underline{x_{i}}, 1-\overline{x_{i}}\right)$.

\section{Proposition 4.3}


Let $A$ be a fuzzy rough set, then $\forall x_{i} \in A$ value of information measure (4.1) is equal to one i.e. it assumes a unique maximum value for $\underline{x_{i}}=0.5$ and $\overline{x_{i}}=0.5$.

\section{Proposition 4.4}

Let $A, B$ be fuzzy rough sets, then $\forall x_{i} \in A$ and $x_{j} \in B$,value of information measure for $A$ is greater than or equal to the value of information measure for $B$ when $B$ is sharper or crisper than A that is $B \leq A$ for $x_{i} \leq 0.5\left(\overline{x_{i}} \leq 0.5\right)$ and $B \geq A$ for $x_{i} \geq 0.5\left(\underline{x_{i}} \geq 0.5\right)$.

These propositions can be proved by following the same procedure as described in previous section 3.

\subsection{Application and Illustration}

In this section application of fuzzy rough information measures (3.4) and (4.1) is illustrated with two examples.

\section{Example 4.1.}

Let us consider the case of choice of restaurants in a particular city by the customers due to several factors such as quality of food, service provide, behaviour of staff etc. It is clear from customer's behaviour that they are not happy with any one restaurant. They prefer different restaurants on the basis of their taste. This whole situation is represented in the form of fuzzy rough set as follows:

Suppose $R=\left\{r_{1}, r_{2}, r_{3}, r_{4}, r_{5}\right\}$ be the set of restaurants in a particular city and $C=\left\{c_{1}, c_{2}, c_{3}, c_{4}, c_{5}, c_{6}\right\}$ be the set of attributes on basis of the taste of customers as $c_{1}=$ interior decoration, $c_{2}=$ best possible value of customers money, $c_{3}=$ location, $c_{4}=$ behaviour of staff, $c_{5}=$ quality of food and $c_{6}=$ offer available.

The aim is to find out which restaurant is best in term of which attribute by using the information given by customers. The information available is model in the form of fuzzy rough set and represented in tabular form as given below:

Table 4.1: Fuzzy Rough Set

\begin{tabular}{|c|c|c|c|c|c|c|}
\hline $\mathrm{C}$ & $c_{1}$ & $c_{2}$ & $c_{3}$ & $c_{4}$ & $c_{5}$ & $c_{6}$ \\
\hline$r_{1}$ & $(.1, .5)$ & $(.2, .6)$ & $(.4, .8)$ & $(.7, .9)$ & $(.6, .8)$ & $(.4, .6)$ \\
\hline$r_{2}$ & $(.2, .3)$ & $(.1, .3)$ & $(.4, .9)$ & $(.6, .8)$ & $(.5, .6)$ & $(.8, .8)$ \\
\hline$r_{3}$ & $(.1, .5)$ & $(.3, .5)$ & $(.5, .7)$ & $(.3, .8)$ & $(.6, .6)$ & $(.4, .9)$ \\
\hline$r_{4}$ & $(.5, .7)$ & $(.1, .5)$ & $(.3, .8)$ & $(.3, .7)$ & $(.1, .3)$ & $(.5, .8)$ \\
\hline$r_{5}$ & $(.4, .6)$ & $(.5, .7)$ & $(.3, .5)$ & $(.7, .9)$ & $(.4, .5)$ & $(.5, .6)$ \\
\hline
\end{tabular}

From above table we find the uncertainty among restaurants and given attribute by using information measure for fuzzy rough set (4.1) as $E_{\log }(R: C)=0.626786$.

Now we find out the uncertainties among restaurants and attributes by using information measure (3.4) for fuzzy rough values which are given below in table 4.2: 
Table 4.2: Uncertainties among Restaurants and Attributes

\begin{tabular}{|c|c|c|c|c|c|c|}
\hline $\mathrm{C}$ & $c_{1}$ & $c_{2}$ & $c_{3}$ & $c_{4}$ & $c_{5}$ & $c_{6}$ \\
\hline$e_{\log \left(r_{1}\right)}$ & 0.678072 & 0.678072 & 0.678072 & $\mathbf{0 . 4 8 5 4 2 7}$ & 0.678072 & 0.847997 \\
\hline$e_{\log \left(r_{2}\right)}$ & 0.584963 & 0.584963 & 0.584963 & 0.678072 & 0.926 & $\mathbf{0 . 4 8 5 4 2 7}$ \\
\hline$e_{\log \left(r_{3}\right)}$ & 0.678072 & 0.847997 & 0.847997 & 0.584963 & 0.847997 & $\mathbf{0 . 5 8 4 9 6 3}$ \\
\hline$e_{\log \left(r_{4}\right)}$ & 0.847997 & 0.678072 & 0.584963 & 0.678072 & $\mathbf{0 . 4 8 5 4 2 7}$ & 0.765535 \\
\hline$e_{\log \left(r_{5}\right)}$ & 0.847997 & 0.847997 & 0.847997 & $\mathbf{0 . 4 8 5 4 2 7}$ & 0.926 & 0.926 \\
\hline
\end{tabular}

Comparing the uncertainty $E_{\log }(R: C)$ with those in table 4.2 and choosing the uncertainty less than or equal to $E_{\log }(R: C)$ we can conclude that restaurants 1 and 5 are good in terms of attribute $c_{4}$ i.e. behaviour of staff of restaurants 2 and 3 are good in terms of attribute $c_{6}$ i.e. offer available and restaurant 4 is good in terms of attribute $c_{5}$ i.e. quality of food.

\section{Example 4.2.}

Covid-19 pandemic has transformed individual lives as well as social life on global scale. The most effected sector by this virus is healthcare. Healthcare personnel have faced a significant higher risk of infection, particularly in the early stages of the outbreak. On an average it takes 5-6 days for symptoms to appear when someone is infected with the corona virus. Symptoms of covid-19 are divided into three categories as

\section{Most Common Symptoms}

- Dry Cough

- Tiredness

- Fever

\section{Less Common Symptoms}

- Headache

- Diarrhoea

- Sore throat

- Rash on Skin

- Discolouration of Finger

- Aches and Pain

- Conjunctivitis

- Loss of taste or Smell

\section{Serious Symptoms}

- Chest Pain

- Loss of Movement or Speech

- Shortness of breath or Difficulty in Breathing

If you have serious type of symptoms then immediate medical attention is required. For most common and mild symptoms, people should stay at home and consult doctor for precaution and cure.

Here we make use of fuzzy rough information measure to detect the type of symptoms in patient for covid-19 and suggest whether patient requires immediate medical attention or not. To see the 
application of the fuzzy rough information measure a hypothetical case study is framed as follows:

Let $A=\left\{A_{1}, A_{2}, A_{3}, A_{4}\right\}$ is the set of patients under study and

$T=\left\{T_{1}=\right.$ Fever,$T_{2}=$ Headache, $T_{3}=$ Chest pain,$T_{4}=$ Difficulty in breathing, $T_{5}=$ Loss of speech $\}$ are the symptoms of the patients. Symptoms $T_{3}, T_{4}, T_{5}$ are the serious type of symptoms and if they occur then immediate treatment is required. The data for the relation between patients and symptoms is given hypothetically as

Table 4.3: Fuzzy Rough Set for Patients and Symptoms

\begin{tabular}{|c|c|c|c|c|c|}
\hline $\mathbf{T}$ & $T_{1}$ & $T_{2}$ & $T_{3}$ & $T_{4}$ & $T_{5}$ \\
\hline$A_{1}$ & {$[0.0,0.4]$} & {$[0.6,0.9]$} & {$[0.1,0.5]$} & {$[0.3,0.7]$} & {$[0.5,0.7]$} \\
\hline$A_{2}$ & {$[0.3,0.5]$} & {$[0.2,0.6]$} & {$[0.2,0.3]$} & {$[0.5,0.9]$} & {$[0.6,0.9]$} \\
\hline$A_{3}$ & {$[0.7,0.8]$} & {$[0.0,0.9]$} & {$[0.2,0.3]$} & {$[0.1,0.2]$} & {$[0.7,1]$} \\
\hline$A_{4}$ & {$[0.9,1]$} & {$[0.3,0.8]$} & {$[0.3,0.4]$} & {$[0.2,0.6]$} & {$[0.5,0.5]$} \\
\hline
\end{tabular}

Using fuzzy rough information measure (3.4), the uncertainties for patients and symptoms are given below in table 4.4:

Table 4.4: Uncertainties among Patients and Symptoms

\begin{tabular}{|c|c|c|c|c|c|}
\hline $\mathbf{T}$ & $T_{1}$ & $T_{2}$ & $T_{3}$ & $T_{4}$ & $T_{5}$ \\
\hline$A_{1}$ & 0.4855 & 0.585 & 0.6781 & 0.6781 & 0.848 \\
\hline$A_{2}$ & 0.848 & 0.6781 & 0.585 & 0.6781 & 0.585 \\
\hline$A_{3}$ & 0.585 & 0.1375 & 0.585 & 0.3785 & 0.3785 \\
\hline$A_{4}$ & 0.1375 & 0.585 & 0.7656 & 0.6781 & 1 \\
\hline
\end{tabular}

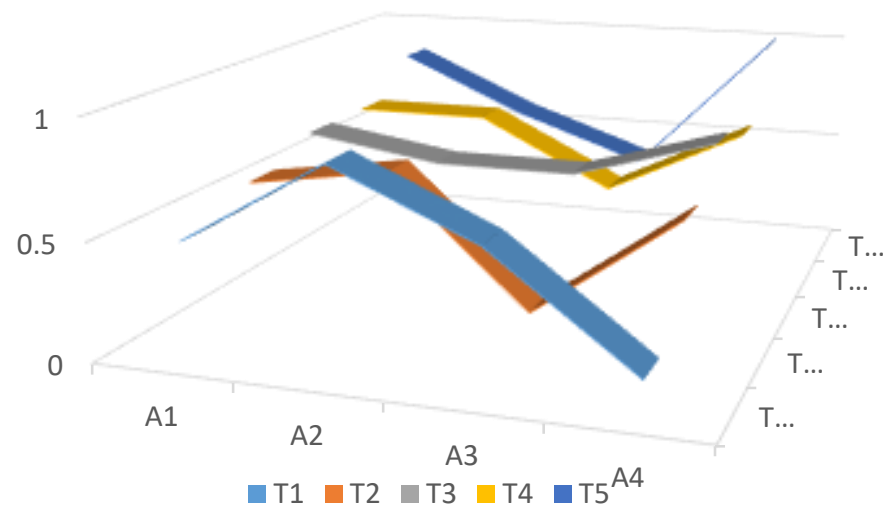

Fig 1: Uncertainties among Patients and Symptoms 
The uncertainty for complete set using fuzzy rough information measure (4.1) is $E_{\log }(A ; T)=0.593980$. Now, compare the uncertainty $E_{\log }(A ; T)$ with those in table 4.4 , if uncertainties for symptoms $T_{3}, T_{4}, T_{5}$ together are greater than $E_{\log }(A ; T)$, then the patient is suffering from serious symptoms of covid-19. From table 4.4 it is clear that patients $A_{1}$ and $A_{4}$ are suffering from serious symptoms and need immediate medical treatment, while patients $A_{2}$ and $A_{3}$ are less effected by virus and can take home remedies to cure themselves.

\section{Weighted Information MEASURE FOR FuZzY ROUGH SET}

In this section weighted information measure for fuzzy rough set is proposed corresponding to measure (3.4) and an application is presented.

Let us consider a fuzzy rough $\operatorname{set}^{\prime} A^{\prime}, \forall x_{i} \in A$ and $w_{i} \in[0,1]$ is the weight for element $x_{i}$ of $A$, then weighted fuzzy rough information measure for $\mathrm{A}$ is given below:

$\forall x_{i} \in A, w_{i} \in[0,1]$ and $x_{i}=\left(\underline{x_{i}}, \overline{x_{i}}\right)$.

(5.1) is called weighted fuzzy rough information measure. Next, we state some propositions for the validity of (5.1).

\section{Proposition 5.1}

Let $A$ be a fuzzy rough set, then $\forall x_{i} \in A$ value of weighted information measure (5.1) is equal to zero for $x_{i}=0$ or 1 and $\overline{x_{i}}=0$ or 1 i.e. for A to be a crisp set.

\section{Proposition 5.2}

Let $A$ be a fuzzy rough set, then $\forall x_{i} \in A$ the value of weighted information measure (5.1) is equal to the value of its complement, where $A^{C}=\left(1-\underline{x_{i}}, 1-\overline{x_{i}}\right)$.

\section{Proposition 5.3}

Let $A$ be a fuzzy rough set, then $\forall x_{i} \in A$ the value of weighted information measure (5.1) is equal to one i.e. it assumes a unique maximum value for $\underline{x_{i}}=0.5$ and $\overline{x_{i}}=0.5$.

\section{Proposition 5.4}

Let $A, B$ be fuzzy rough sets, then $\forall x_{i} \in A$ and $x_{j} \in B$, the value of weighted information measure for $A$ is greater than or equal to the value of weighted information measure for $B$ when $B$ is sharper or crisper than $\mathrm{A}$ that is $B \leq A$ for $x_{i} \leq 0.5\left(\overline{x_{i}} \leq 0.5\right)$ and $B \geq A$ for $x_{i} \geq$ $0.5\left(\underline{x_{i}} \geq 0.5\right)$.

It may noted that the above proposition hold good obviously, so the weighted fuzzy information measure is a valid measure. 


\subsection{Application for Illustration}

For illustration purpose the data set of example 4.1 is considered in the following table:

Table 5.1: Fuzzy Rough Set

\begin{tabular}{|c|c|c|c|c|c|c|}
\hline $\begin{array}{l}\mathrm{C} \\
\mathrm{R}\end{array}$ & $c_{1}$ & $c_{2}$ & $c_{3}$ & $c_{4}$ & $c_{5}$ & $c_{6}$ \\
\hline$r_{1}$ & $(.1, .5)$ & $(.2, .6)$ & $(.4, .8)$ & $(.7, .9)$ & $(.6, .8)$ & $(.4, .6)$ \\
\hline$r_{2}$ & $(.2, .3)$ & $(.1, .3)$ & $(.4, .9)$ & $(.6, .8)$ & $(.5, .6)$ & $(.8, .8)$ \\
\hline$r_{3}$ & $(.1, .5)$ & $(.3, .5)$ & $(.5, .7)$ & $(.3, .8)$ & $(.6, .6)$ & $(.4, .9)$ \\
\hline$r_{4}$ & $(.5, .7)$ & $(.1, .5)$ & $(.3, .8)$ & $(.3, .7)$ & $(.1, .3)$ & $(.5, .8)$ \\
\hline$r_{5}$ & $(.4, .6)$ & $(.5, .7)$ & $(.3, .5)$ & $(.7, .9)$ & $(.4, .5)$ & $(.5, .6)$ \\
\hline
\end{tabular}

The uncertainties calculated on applying fuzzy rough information measure (3.4) are given in table 5.2 .

Table 5.2: Uncertainties among Restaurants and Attributes

\begin{tabular}{|c|c|c|c|c|c|c|}
\hline $\mathrm{C}$ & $c_{1}$ & $c_{2}$ & $c_{3}$ & $c_{4}$ & $c_{5}$ & $c_{6}$ \\
$\mathrm{R}$ & & & & & & \\
\hline$e_{\log }\left(r_{1}\right)$ & 0.678072 & 0.678072 & 0.678072 & 0.485427 & 0.678072 & 0.847997 \\
\hline$e_{\log }\left(r_{2}\right)$ & 0.584963 & 0.584963 & 0.584963 & 0.678072 & 0.926 & 0.485427 \\
\hline$e_{\log }\left(r_{3}\right)$ & 0.678072 & 0.847997 & 0.847997 & 0.584963 & 0.847997 & 0.584963 \\
\hline$e_{\log }\left(r_{4}\right)$ & 0.847997 & 0.678072 & 0.584963 & 0.678072 & 0.485427 & 0.765535 \\
\hline$e_{\log }\left(r_{5}\right)$ & 0.847997 & 0.847997 & 0.847997 & 0.485427 & 0.926 & 0.926 \\
\hline
\end{tabular}

Now, the weights corresponding to each element of table 5.1 are given below:

Table 5.3: Weights Corresponding to Each Element of Fuzzy Rough Set

\begin{tabular}{|l|l|l|l|l|l|}
\hline 0.53 & 0.62 & 0.55 & 0.82 & 0.92 & 0.87 \\
\hline 0.84 & 0.80 & 0.66 & 0.45 & 0.33 & 0.23 \\
\hline 0.16 & 0.25 & 0.39 & 0.64 & 0.87 & 0.33 \\
\hline 0.29 & 0.36 & 0.46 & 0.47 & 0.78 & 0.63 \\
\hline 0.13 & 0.85 & 0.74 & 0.53 & 0.24 & 0.82 \\
\hline
\end{tabular}

Using weighted fuzzy rough information measure (5.1), the weighted uncertainties for fuzzy rough values are

Table 5.4: Uncertainties Using Weighted Fuzzy Rough Information Measure (5.1)

\begin{tabular}{|c|c|c|c|c|c|c|}
\hline${ }_{R} C$ & $c_{1}$ & $c_{2}$ & $c_{3}$ & $c_{4}$ & $c_{5}$ & $c_{6}$ \\
\hline$h_{\log }\left(r_{1}\right)$ & 0.3594 & 0.4204 & 0.3730 & 0.3980 & 0.6239 & 0.7378 \\
\hline $\boldsymbol{h}_{\log }\left(\boldsymbol{r}_{2}\right)$ & 0.4914 & 0.468 & 0.3861 & 0.3051 & 0.3056 & 0.1116 \\
\hline$h_{\text {log }}\left(r_{3}\right)$ & 0.1085 & 0.212 & 0.3307 & 0.3744 & 0.7378 & 0.1931 \\
\hline$h_{\log }\left(r_{4}\right)$ & 0.2459 & 0.2441 & 0.2691 & 0.3187 & 0.3786 & 0.4823 \\
\hline$h_{\log }\left(r_{5}\right)$ & 0.1102 & 0.7208 & 0.6275 & 0.2573 & 0.2222 & 0.7593 \\
\hline
\end{tabular}


Weighted uncertainty for complete fuzzy rough set is $H_{\log }(A ; T)=0.6988$.After assigning the weights it can be noted that the decision regarding restaurants is changed. All the restaurants are up to the expectations of customers except restaurants $r_{1}, r_{3}$ and $r_{5}$, particularly for attributes $c_{6}$ of restaurant $r_{1}, c_{5}$ of restaurant $r_{3}$ and $c_{2}, c_{6}$ of restaurant $r_{5}$. Since, the uncertainties corresponding to these attributes are greater than $H_{\text {log }}(A ; T)$.

\section{COMPARISON WITH OTHER FUZZY ROUGH INFORMATION MEASURES}

In this section information measure (3.4) and (4.1) are compared with the existing fuzzy rough information measures defined and studied by Sharma and Gupta [21].

Let ' $A$ ' be a rough set then, sine trigonometric information measures for fuzzy rough values of set ' $A$ ' and for fuzzy rough set ' $A$ ' are written respectively as given below:

$e_{\sin }(x)=\sin \left[\frac{\pi}{2}\left(1-\frac{1}{2}(|2 \underline{x}-1|+|2 \bar{x}-1|)\right)\right]$, for every element $x \in A$,

and $E_{\text {sin }}(x)=\frac{1}{n} \sum_{i=1}^{n} \sin \left[\frac{\pi}{2}\left(1-\frac{1}{2}\left(\left|2 \underline{x_{i}}-1\right|+\left|2 \overline{x_{i}}-1\right|\right)\right)\right]$, for the whole set A.

Similarly, the cosine and tangent trigonometric information measures for fuzzy rough values and for fuzzy rough set respectively are

$e_{\cos }(x)=\cos \left[\frac{\pi}{4}(|2 \underline{x}-1|+|2 \bar{x}-1|)\right]$, for every element $x \in A$,

$E_{\cos }(x)=\frac{1}{n} \sum_{i=1}^{n} \cos \left[\frac{\pi}{4}\left(\left|2 \underline{x_{i}}-1\right|+\left|2 \overline{x_{i}}-1\right|\right)\right]$, for the whole set A.

$e_{\tan }(x)=\tan \left[\frac{\pi}{4}\left(1-\frac{1}{2}(|2 \underline{x}-1|+|2 \bar{x}-1|)\right)\right]$, for every element $x \in A$,

and

$E_{\tan }(x)=\frac{1}{n} \sum_{i=1}^{n} \tan \left[\frac{\pi}{4}\left(1-\frac{1}{2}\left(\left|2 \underline{x_{i}}-1\right|+\left|2 \overline{x_{i}}-1\right|\right)\right)\right]$, for the whole set A.

The uncertainties for fuzzy rough values in example 4.1 are computed using the above trigonometric fuzzy rough information measures as given in table 6.1. 
Table 6.1: Uncertainties using Trigonometric FRI Measures

\begin{tabular}{|c|c|c|c|c|c|c|}
\hline $\mathrm{R}$ & $c_{1}$ & $c_{2}$ & $c_{3}$ & $c_{4}$ & $c_{5}$ & $c_{6}$ \\
\hline$e_{\sin }\left(r_{1}\right)$ & .8090 & .8090 & .8090 & .5878 & .8090 & .9511 \\
\hline$e_{\cos }\left(r_{1}\right)$ & .8090 & .8090 & .8090 & .5878 & .8090 & .9511 \\
\hline$e_{\text {tan }}\left(r_{1}\right)$ & .5095 & .5095 & .5095 & .3249 & .5095 & .7265 \\
\hline$e_{\sin }\left(r_{2}\right)$ & .7071 & .5878 & .7071 & .8090 & .9877 & .5878 \\
\hline $\boldsymbol{e}_{\cos }\left(\boldsymbol{r}_{2}\right)$ & .7071 & .5878 & .7071 & .8090 & .9877 & .5878 \\
\hline$e_{t a n}\left(r_{2}\right)$ & .4142 & .3249 & .4142 & .5095 & .8541 & .3249 \\
\hline$e_{\sin }\left(r_{3}\right)$ & .8090 & .9511 & .9511 & .7071 & .9511 & .7071 \\
\hline$e_{\cos }\left(r_{3}\right)$ & .8090 & .9511 & .9511 & .7071 & .9511 & .7071 \\
\hline$e_{\text {tan }}\left(r_{3}\right)$ & .5095 & .7265 & .7265 & .4242 & .7265 & .4142 \\
\hline$e_{\sin }\left(r_{4}\right)$ & .9511 & .8090 & .7071 & .8090 & .5878 & .8910 \\
\hline$e_{\cos }\left(r_{4}\right)$ & .9511 & .8090 & .7071 & .8090 & .5878 & .8910 \\
\hline$e_{\tan }\left(r_{4}\right)$ & .7265 & .5095 & .4142 & .5095 & .3249 & .6128 \\
\hline$e_{\sin }\left(r_{5}\right)$ & .9511 & .9511 & .9511 & .5878 & .9877 & .9877 \\
\hline$e_{\cos }\left(r_{5}\right)$ & .9511 & .9511 & .9511 & .5878 & .9877 & .9877 \\
\hline$e_{t a n}\left(r_{5}\right)$ & .7265 & .7265 & .7265 & .3249 & .8541 & .8541 \\
\hline
\end{tabular}

The uncertainties calculated using the trigonometric fuzzy rough information measures for fuzzy rough set are $E_{\text {sin }}(R)=0.8136, E_{\text {cos }}(R)=0.8136$ and $E_{\text {tan }}(R)=0.55862$.

\section{Uncertainties Using Trigonometric Information Measures}

1.2

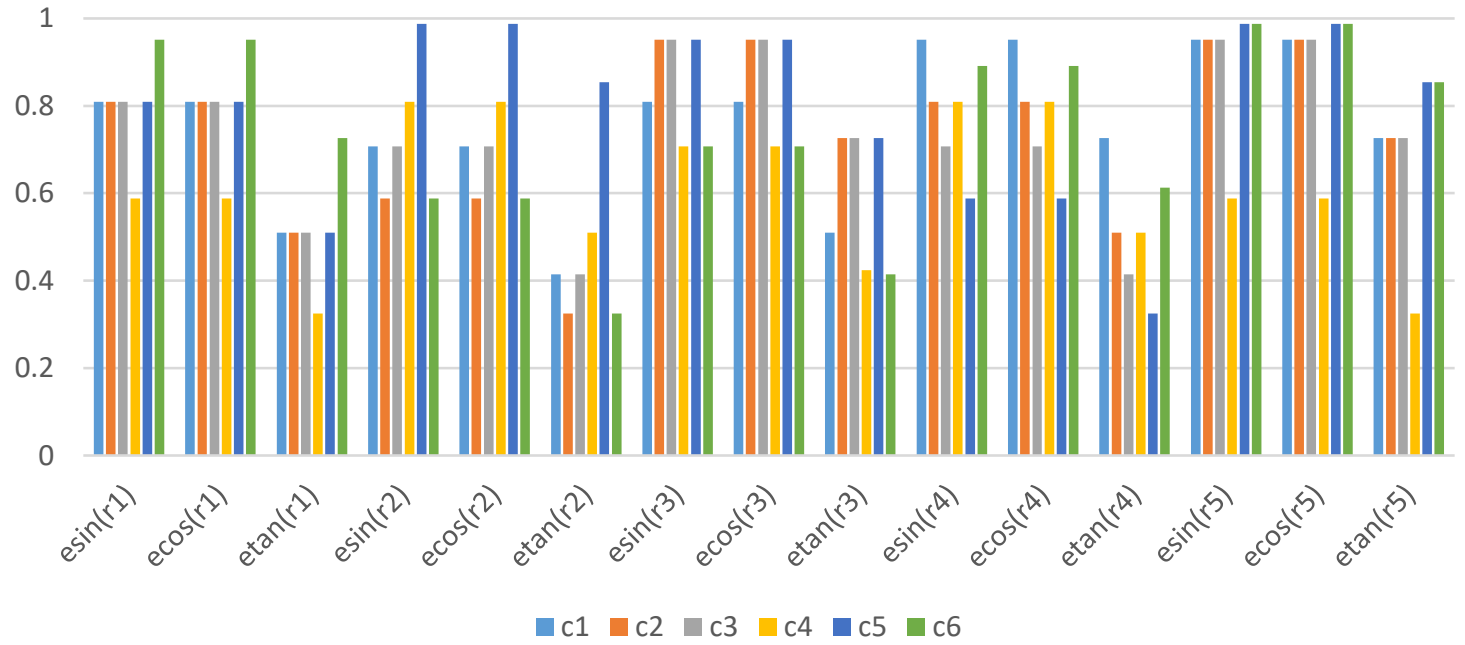

Fig 2. Uncertainties Using Trigonometric FRI measures

Next, the uncertainties calculated using information measure (3.4) for fuzzy rough values of example 4.1 are given below: 
Table 6.2: Uncertainty Using FRI Measure (3.4)

\begin{tabular}{|l|c|c|c|c|c|c|}
\hline $\mathrm{C}$ & $c_{1}$ & $c_{2}$ & $c_{3}$ & $c_{4}$ & $c_{5}$ & \multicolumn{1}{c|}{$c_{6}$} \\
\hline$e_{\log }\left(r_{1}\right)$ & 0.678072 & 0.678072 & 0.678072 & $\mathbf{0 . 4 8 5 4 2 7}$ & 0.678072 & 0.847997 \\
\hline$e_{\log }\left(r_{2}\right)$ & 0.584963 & 0.584963 & 0.584963 & 0.678072 & 0.926 & $\mathbf{0 . 4 8 5 4 2 7}$ \\
\hline$e_{\log }\left(r_{3}\right)$ & 0.678072 & 0.847997 & 0.847997 & 0.584963 & 0.847997 & $\mathbf{0 . 5 8 4 9 6 3}$ \\
\hline$e_{\log }\left(r_{4}\right)$ & 0.847997 & 0.678072 & 0.584963 & 0.678072 & $\mathbf{0 . 4 8 5 4 2 7}$ & 0.765535 \\
\hline$e_{\log }\left(r_{5}\right)$ & 0.847997 & 0.847997 & 0.847997 & $\mathbf{0 . 4 8 5 4 2 7}$ & 0.926 & 0.926 \\
\hline
\end{tabular}

Now, uncertainty computed by information measure (4.1) for fuzzy rough $\operatorname{set}$ is $E_{\log }(R: C)=$ 0.626786 .

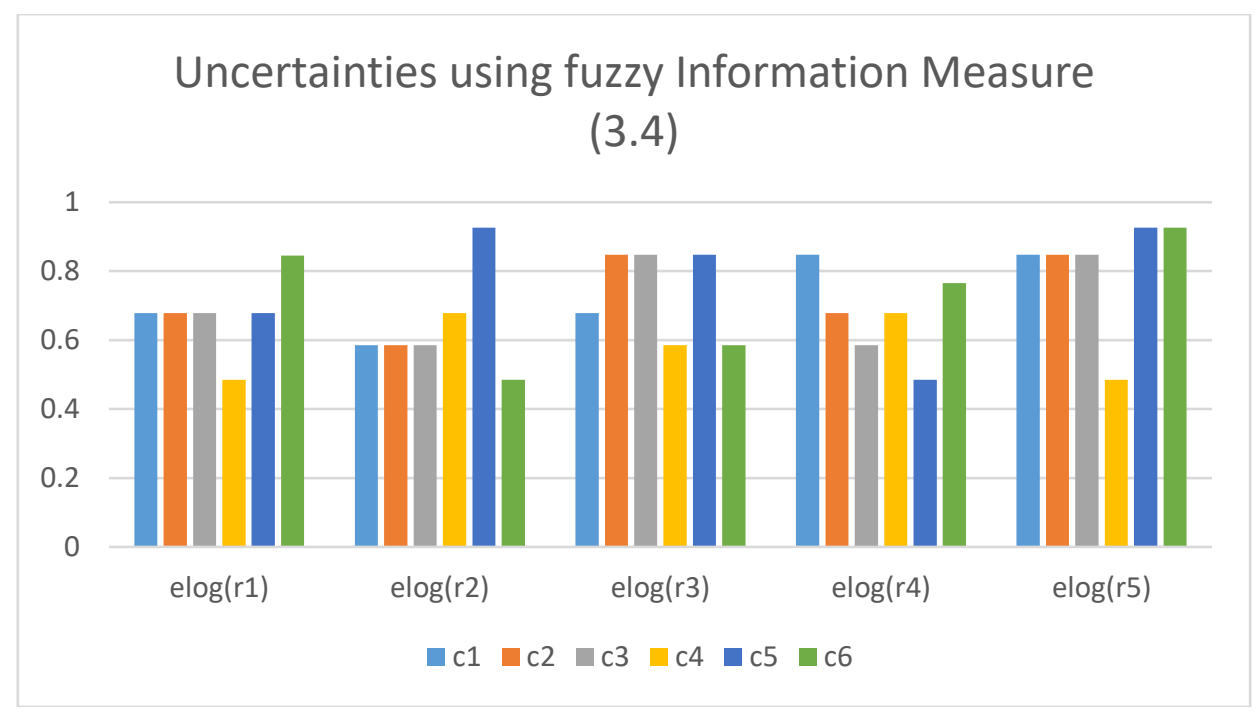

Fig 3: Uncertainties Using fuzzy rough Information Measure (3.4)

From the table 6.1 and 6.2 and from the graphs it may be noted that the uncertainties calculated on applying information measure (3.4) are much less than that of sine and cosine trigonometric information measures studied by Sharma and Gupta [21]. Thus, our information measure is more useful and simple than the trigonometric information measures. However, the uncertainty calculated using sine and cosine measures are almost same.

\section{CONCLUSION}

As we know a right decision can change one's life, so it is important to study decision making methods for solving the problems of our daily life and fuzzy rough set theory is one of the choices. Fuzzy rough set is the hybridization of rough set and fuzzy set which had been widely used to deals with complex data containing different types of uncertainties. In this paper a logarithmic information measure for fuzzy rough values is proposed and verified axiomatically. Logarithmic information measure for fuzzy rough set and weighted logarithmic fuzzy rough information measure are also defined with their application in decision making problem. The Proposed fuzzy rough information measure is compared with other existing trigonometric fuzzy rough information measures and it is proved that our information measure is better and simple. 
The research on application of fuzzy rough set theory can be extended in studying data dimension reduction technique and decision making in design of experiment. A new form of rough set that is hyper rough set can be defined. Hyper rough set is completely a new idea in the area of rough set theory. In future researchers can put their attention towards defining new information measures by combining logarithmic and trigonometric functions as combination of two functions overcome the shortcoming of one another and provide better result than single one.

\section{CONFLICT OF INTEREST}

There is no conflict of Interest among the authors.

\section{REFERENCES}

[1] Banerjee M. and Pal S.K. Roughness of a Fuzzy Set. Informatics and Computer Sciences, 93(1996), 235-246.

[2] Chengyi Z, Pingan D. and Haiyan F. On Measures of Similarity between Fuzzy Rough Sets. International Journal of Pure and Applied Mathematics, 10(2004), 451-460.

[3] De Luca and Termini S. A Definition of a Non-Probabilistic Entropy in the Setting of Fuzzy Sets Theory. Fuzzy Sets and Systems, 52(1972), 305-318.

[4] Dubois D. and Prade H. Rough Fuzzy Sets and Fuzzy Rough Sets. International Journal of General Systems, 17(1990), 191-209.

[5] Gupta P. and Sheoran A. Some New Parametric Fuzzy Entropies. International Journal of Fuzzy Mathematics and Systems, 4(2014), 293-298.

[6] Hooda D.S. and Divya Jain. Sub additive Measures of Fuzzy Information. Journal of Reliability and Statistical Studies, 2(2009), 39-52.

[7] Hooda D.S. and Bajaj R.K. "Useful"-Fuzzy Measure of Information, Integrated Ambiguity and Directed Divergence. International Journal of General Systems, Taylor and Francis, First Article, (2010), 10-12.

[8] Hooda D.S. and Raich V. Fuzzy Information Measures with Applications. Alpha Science, International LTD., 7200, The Quorum, Oxford Business Park North Garsington Road, Oxford OX4 2JZ, U.K. ISBN- 978-1-84265-934-2(2015).

[9] Jabar A.L. and Rashid T.A. Combining Fuzzy Rough Set with Salient Features for HRM Classification. Conference- $15^{\text {th }}$ IEEE International Conference on Computer and Information Technology (CIT-2015), At. Liverpool, England, U.K., 244-251. DOI: 10.1109/CIT/1UCC/DASC/PICOM.2015.35.

[10] Kapur T.N. Measure of Fuzzy Information. New Delhi Mathematical Sciences of Trust Society (1997).

[11] Kosko B. Fuzzy Entropy and Conditioning. Information Sciences, 40(1986), 165-174.

[12] Liu X.C. Entropy Distance Measure and Similarity Measure of Fuzzy Sets and Their Relations. Fuzzy Sets and Systems, 52(1992), 305-318.

[13] Nakamura A. Fuzzy Rough Sets. Note on Multiple Valued Logic in Japan, 9(1988), 1-8.

[14] Nanda S. and Majumdar S. Fuzzy Rough Sets. Fuzzy Sets and Systems, 145(1992), 157-160.

[15] Pal N.R. and Pal S.K. Entropy-A New Definition and its Applications. IEEE Transactions on Systems, Man and Cybernetics, 21(1999), 1260-1270.

[16] Pawlak Z. Rough Sets. International Journal of Information and Computational Sciences, 11(1982), 341-356.

[17] Qi N. and Chengyi Z. A New Similarity Measures on Fuzzy Rough Sets. International Journal of Pure and Applied Mathematics, 47(2008), 89-100.

[18] Shannon C.E. A Mathematical Theory of Communication. Bell System Technical Journal, 27(1948), 379-423.

[19] Sharma O., Rani A. and Gupta P. Some Similarity and Distance Measures on Fuzzy Rough Sets and its Applications. International Journal of Engineering Sciences and Mathematics, 6(2017), 85-105.

[20] Sharma O., Tiwari P. and Gupta P. (Communicated). Information Measures for Fuzzy Rough Sets and their Applications in Pattern Recognition and Medical Area. 
[21] Sharma O. and Gupta P. Some Trigonometric Entropies for Fuzzy Rough Set and their Application in Medical Area. International Journal for Research in Engineering Application and Management, 4(2018), 570-578.

[22] Sharma O. Logarithmic Entropy Measures for Fuzzy Rough Set and their Application in Decision Making. International Journal of Fuzzy System and Applications, 9(2020), 80-97.

[23] Skowron A. and Grzym.la- Busse J.W. From Rough Set Theory to Evidence Theory. In Advance in the Dempster-Shafter Theory of Evidence, R.R. Yaeger, M. Fedrizzi and J. Kacprzyk. Eds. Wiley New York, (1994), 193-236.

[24] Sun S. Li. L. and Hu. K. A New Approach to Rough Set Based on Remote Neighbourhood System. Mathematical Problems in Engineering, (2019), 1-8. DOI: https://doi.org/10.1155/2019/8712010.

[25] Verbiest N. Fuzzy Rough and Evolutionary Approaches to Instance Selection. PhD Thesis, Faculty of Sciences, Ghent University, March 2014.

[26] Zadeh L.A. Fuzzy Sets. Information and Control, 8(1965), 338-356.

[27] Zadeh L.A. Fuzzy Sets and Systems. In Proceeding of the Symposium on System Theory, (1965), 2937. Polytechnic Institute of Brooklyn, Brooklyn, NY, USA.

[28] Zhan J. Sun B. and Alcantud J.C. Covering Based Multi-Granulation (I.T)-Fuzzy Rough Set Models and Application in Multi-Attribute Group Decision Making. Information Sciences, 476(2019), 290318. 